\title{
Predicting Diagnosis of Alzheimer's Disease and Related Dementias Using Administrative Claims
}

\author{
Jennifer S. Albrecht, PhD; Maya Hanna, MPH; Dure Kim, PharmD; and Eleanor M. Perfetto, PhD, MS
}

\begin{abstract}
BACKGROUND: Predictive models for earlier diagnosis of Alzheimer's disease and related dementias (ADRD) that rely on variables requiring assessment during an office visit, such as cognitive function, body mass index, or lifestyle factors, may not be broadly applicable, since that level of data may be inaccessible or inefficient.

OBJECTIVE: To build a predictive model for earlier diagnosis of ADRD using only administrative claims data to enhance applicability at the health caresystem level. Building on the strength of this approach and knowledge that health care utilization (HCU) is increased before dementia diagnosis, it was hypothesized that previous HCU history would improve predictive ability of the model.
\end{abstract}

METHODS: We conducted a case-control study using data from the OptumLabs Data Warehouse. ADRD was defined using ICD-9-CM codes and prescription fills for antidementia medications. We included individuals with mild cognitive impairment. Cases aged $\geq 18$ years with a diagnosis between 2011-2014 were matched to controls without ADRD. HCU variables were incorporated into regression models along with comorbidities and symptoms.

RESULTS: The derivation cohort comprised 24,521 cases and 95,464 controls. Final adjusted models were stratified by age. We obtained moderate accuracy (c-statistic $=0.76$ ) for the model among younger (aged $<65$ years) adults and poor discriminatory ability (c-statistic $=0.63$ ) for the model among older adults (aged $\geq 65$ years). Neurological and psychological disorders had the largest effect estimates.

CONCLUSIONS: We created age-stratified predictive models for earlier diagnosis of dementia using information available in administrative claims. These models could be used in decision support systems to promote targeted cognitive screening and earlier dementia recognition for individuals aged $<65$ years. These models should be validated in other cohorts.

J Manag Care Spec Pharm. 2018;24(11):1138-45

Copyright $\odot 2018$, Academy of Managed Care Pharmacy. All rights reserved.

\section{What is already known about this subject}

Earlier diagnosis of dementia may reduce health care utilization due to improved management of comorbid illness and delayed institutionalization, thereby decreasing associated costs.

- Only half of all individuals with dementia receive a diagnosis, and often dementia is recognized only when symptoms become severe. Current predictive models for earlier diagnosis of dementia that rely on variables requiring assessment during an office visit may not be broadly applicable at the health-system level.

\section{What this study adds}

Using only administrative claims, predictive models were created to estimate the odds of a diagnosis of dementia in 2 years.

The algorithm used in this study is logistically practical for lowburden implementation for a larger provider, such as an integrated delivery system or insurance plan level.

Best accuracy was observed among individuals aged $<65$ years.

1 he number of persons living with Alzheimer's disease in the United States exceeded 5.5 million in 2017. ${ }^{1}$ Due to the aging population, the number of individuals with Alzheimer's disease and related dementias (ADRD) is projected to double by $20500^{1,2}$ Furthermore, costs of ADRD to society are substantial, with total costs of health care for individuals with ADRD in 2017 estimated at $\$ 259$ billion. $^{1}$

Early recognition of cognitive decline is essential to rule out reversible causes of dementia and may help to delay symptom onset. It also benefits patients and caregivers by providing time to prepare, mentally and financially, for the future. ${ }^{1,3}$ Earlier diagnosis of ADRD may reduce health care utilization (HCU) because of improved management of comorbid illness and delayed institutionalization, thereby decreasing associated costs. ${ }^{1}$ In addition, interventions to delay onset of symptoms must be targeted toward earlier stages of disease. Nonetheless, only half of all individuals with ADRD receive a diagnosis, and often ADRD is recognized only when symptoms become severe. ${ }^{4}$ Primary care providers are most likely to see patients with early signs of ADRD yet may have difficulty recognizing the signs of cognitive impairment during a brief office visit or are reluctant to assign the diagnosis. ${ }^{4}$ Mass cognitive screening is simply not practical or efficient with current tools. ${ }^{5,6}$

To aid in identification of individuals at increased risk of developing ADRD, models have been developed for short- (4-6 years) and long-term (10-40 years) risk prediction. ${ }^{7-13}$ These models have been used to (a) create risk scores that help categorize individuals by level of risk or (b) discriminate between those with and without dementia with moderate accuracy (area under the curve ranging from $0.60-0.78) .^{7-13}$ Targeted application of models could be used in specific populations for efficient and low-cost screening to facilitate earlier diagnosis of ADRD, especially if further refined. 
Models that rely on variables requiring assessment during an office visit, such as cognitive function, body mass index, or lifestyle factors, may not be broadly applicable, since that level of data may be inaccessible or inefficient. In contrast, models that rely on health information that is more generally available, such as claims submitted for reimbursement, may offer an efficient and practical alternative suitable to high-throughput of patient information. To enhance applicability at the health care-system level, the objective of this study was to build a predictive model for earlier diagnosis of ADRD using only administrative claims data. Building on the strength of this approach and knowledge that HCU is increased before dementia diagnosis, it was hypothesized that previous HCU history would improve predictive ability of the model. ${ }^{14-16}$

\section{Methods}

This project was a partnership between the University of Maryland and OptumLabs, which is an open, collaborative research and innovation center founded in 2013 as a partnership between Optum and Mayo Clinic. OptumLabs convened a technical advisory panel to provide input and guidance on its Alzheimer's Disease Initiative, which supported this work. We relied on the panel for clinical guidance throughout the project.

\section{Study Sample}

A case-control study was conducted using data from the OptumLabs Data Warehouse (OLDW), which includes deidentified claims data for privately insured and Medicare Advantage enrollees in a large, private U.S. health plan and currently covers 125 million lives. The database contains longitudinal health information on enrollees, representing a diverse mixture of ages, ethnicities, and geographical regions across the United States and is broadly representative of the general commercial/ Medicare Advantage market in the geographic area where it operates. The health plan provides comprehensive full insurance coverage for physician, hospital, and prescription drug services. In the OLDW, anyone aged $\geq 89$ years is assigned an age of 89 years to maintain patient confidentiality. Since this study involved analysis of preexisting, deidentified data, it was exempt from institutional review board approval.

Previous studies conducted using administrative claims data have largely excluded individuals with mild cognitive impairment and those with antidementia medication use but no dementia diagnosis. ${ }^{17-20}$ We based our definition of dementia on these studies but broadened it with guidance from the OptumLabs technical advisory panel. Individuals aged $\geq 18$ years with a diagnosis of ADRD between January 2011 and June 2014 were eligible for the study.

ADRD was defined using International Classification of Disease, Ninth Revision, Clinical Modification (ICD-9-CM) codes 331.0 (Alzheimer's disease); 331.83 (mild cognitive impairment [MCI]); 331.82 (Lewy-body associated dementia [LBD]); 331.1x (fronto-temporal dementia [FTD]); 290.4x (vascular dementia [VD]); and 290.0x-290.3x, 290.8x, 290.9x, 294.10, 294.11, 294.20, 294.21, and 797 (nonspecific dementias [NSD]). To increase sensitivity of the dementia definition, prescription data were searched for use of antidementia medications (tacrine, donepezil, rivastigimine, galantamine, and memantine/donepezil). The first dementia diagnosis or fill for an antidementia medication following a 36-month continuous insurance enrollment "clean" period with no dementia diagnosis was considered the index date.

In addition to requiring 36 months of continuous health insurance enrollment before the index date (earliest date of study data was January 2008), we also required 6 months of enrollment after the index date (through December 2014) to search for confirmation diagnoses. A confirmation ADRD diagnosis or antidementia prescription was required within 6 months of the index date to increase specificity of our dementia criteria. The confirmation ADRD diagnosis or antidementia prescription did not have to be the same as the index diagnosis or fill for an antidementia medication. If the index diagnosis occurred during hospitalization, we waited 2 weeks before searching for the confirmation diagnosis to ensure that confirmation took place outside the hospitalization.

Individuals were excluded if they had a diagnosis for nutritional deficiencies or alcohol or substance dependency within 3 months of the index date because these disorders can induce cognitive impairment related to mechanisms other than what we were trying to model. We also excluded those with a hospice claim during the 6 months previous to the index date to avoid modeling the end of life rather than dementia diagnosis.

Cases were matched 1:4 to controls with no history of dementia or antidementia medication use during the 36 months pre- or 6 months postmatched index date (2011-2014). Controls were frequency matched on age, sex, and U.S. Census region and assigned the same index date as cases for the purposes of determining health insurance enrollment. The same continuous enrollment and exclusion criteria were applied after matching to the controls, which resulted in the loss of some controls.

\section{Dementia Categories}

Based on our cohort definition criteria, we initially created 7 dementia categories: Alzheimer's disease, MCI, LBD, FTD, VD, NSD, and drug use only. However, the index and confirmation diagnoses often differed, and many individuals received several diagnoses, which prompted the creation of a set of clinically guided rules to facilitate categorization of individuals into dementia diagnostic categories.

\section{Health Care Utilization}

Counts were created of all-cause HCU events occurring in the third year before the index date. (i.e., index date occurs in month 0 , counts are from months -36 to -25$)$. Events included 


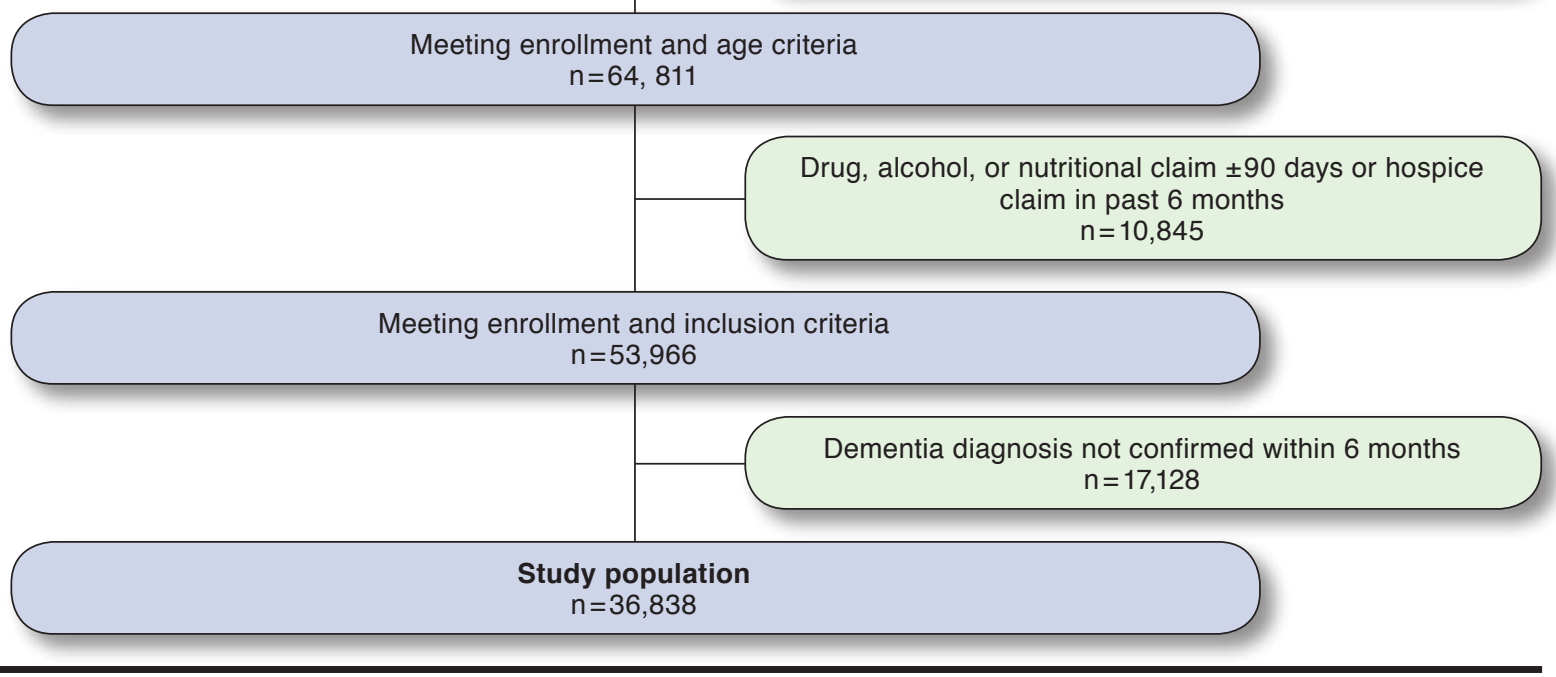

hospitalization; outpatient; long-term care; ancillary services (lab tests, radiology, and medications); and any HCU from any category. The distribution of HCU counts tended to be left-skewed. Based on the distribution, counts were either dichotomized at the median or categorized based on quartiles to create categorical variables. In addition, we also examined disease-specific HCU. Based on initial bivariate analyses, the following diseases were selected because they had the largest proportional differences between cases and controls: diabetes, psychiatric disorders, anemia, stroke, Parkinson's disease, heart failure, and chronic obstructive pulmonary disease.

\section{Additional Covariates}

We searched all claim types for ICD-9-CM codes representing diagnoses for comorbidities or symptoms of interest during the third year before the index date. Comorbidities and symptoms were selected based on associations with dementia or with increased HCU and included atrial fibrillation, depression, hyperlipidemia, Parkinson's disease, and traumatic brain injury. Symptoms included weight loss, coordination difficulties, and memory complaints. We used the entire third year before diagnosis to assess covariates.

\section{Data Analysis}

The study population was randomly divided into 2 cohorts. Two thirds of the population became the derivation cohort, and the remaining third was used as the validation cohort. We compared distributions of demographic, clinical, and utilization variables between cases and controls, and used Student's t-test or the chi-square goodness of fit to test for significant differences. Logistic regression was used, rather than conditional logistic regression, to model the log-odds of case status as a function of matching variables, $\mathrm{HCU}$, and additional covariates. The large sample precluded sparse data problems that might otherwise have necessitated conditional logistic regression, and regular logistic regression facilitated variable selection, as well as assessment of discriminatory ability of the models.

The modeling was conducted in stages-the initial model contained only the matching and HCU variables. Given the large number of covariates, stepwise selection was used to select predictor variables for the final model; forced entry into the model was used for the matching variables. The area under the curve (AUC) was examined to assess the discriminatory ability of the models. Following creation of the final models in the derivation cohort, we ran the models in the validation cohort to determine whether predictive ability was consistent. The critical $P$ value for all analyses was $<0.001$.

Sensitivity analyses were also conducted. We increased the $P$ values used in the stepwise selection process to determine if the additional variables selected improved discrimination. Models were then stratified by different age cut-points and by dementia-specific categories (i.e., $\mathrm{AD}$ and $\mathrm{MCI}$ ). 


\section{Results}

There were 565,292 individuals with a diagnosis of dementia or a prescription fill for an antidementia medication between 2011-2014 (Figure 1). Of these, 64,811 (11\%) were aged $\geq 18$ years, had continuous insurance enrollment 36 months before and 6 months after the index date, and had no diagnosis of dementia before the index date during the 36-month look-back period. Based on exclusion criteria, 10,845 individuals (17\% of 64,811) were excluded. Dementia diagnosis was confirmed within 6 months for 36,838 individuals (68\% of 64,811), creating the final study sample of cases. These individuals were matched 1:4 to controls. We next applied our exclusion criteria (nutritional deficiencies and alcohol or substance dependency within 3 months of the index date and hospice claim during the 6 months before the index date) to the controls. There were 143,419 controls, for a total sample size of 180,257 . This sample was divided into the derivation $(n=119,985)$ and validation $(n=60,272)$ cohorts. The remaining results apply only to the derivation cohort (24,521 cases and 95,464 controls) unless otherwise noted.

Among the cases, there were 2,531 (10\%) individuals with Alzheimer's disease; 3,748 (15\%) with MCI; 136 (0.6\%) with LBD; 102 (0.4\%) with FTD; 1,013 (4\%) with VD; 10,046 (41\%) with NSD, 3,833 (16\%), who were only prescribed drugs and never received a dementia diagnosis; and 3,112 (13\%) with multiple dementia diagnoses (Table 1). Average age of cases was 74.9 (standard deviation [SD] 11.5) years, with a range of 18-85 years. Eighty-eight percent of the sample was aged $\geq 65$ years, and $62 \%$ were female. The majority of the sample came from the South (42\%) and Midwest (35\%) U.S. Census regions. Cases ( $n=24,521)$ differed from controls $(n=95,464)$ by insurance status, with cases more likely to be insured through Medicare Advantage ( $74 \%$ vs. $67 \%, P<0.001)$. HCU differed significantly between cases and controls, with cases more likely to have $>21$ all-cause HCU events per year ( $33 \%$ vs. $22 \%, P<0.001$ ). This higher HCU among cases was observed in every HCU category but was especially prominent in long-term care, inpatient, and outpatient visits.

At the start of the second year before diagnosis, cases had a significantly higher burden of comorbidity than controls, with higher prevalence for 36 of 41 measured comorbidities and symptoms $(P<0.001$ for all). Prevalence of psychiatric disorders such as depression ( $12 \%$ vs. $5 \%, P<0.001)$; anxiety (9\% vs. $5 \%, P<0.001)$; and bipolar disorder ( $2 \%$ vs. $<1 \%, P<0.001)$ was higher in cases than in controls (Table 2). Prevalence of other disorders such as diabetes ( $29 \%$ vs. $23 \%, P<0.001)$; obstructive sleep apnea (11\% vs. 7\%, $P<0.001$ ); and stroke (14\% vs. 9\%, $P<0.001)$ was also higher in cases than in controls. Prevalence of falls ( $2 \%$ vs. $1 \%, P<0.001)$ and memory complaints ( $4 \%$ vs. $<1 \%, P<0.001)$ was higher in cases than in controls.

The final adjusted model for cases status contained 30 predictors, including 3 all-cause HCU variables. Inclusion of disease-specific HCU did not improve discriminatory ability of the model with all-cause utilization, which was poor with an AUC of 0.65 . In the sensitivity analysis, the model was stratified by age categories to determine if accuracy would be improved. The model performed better in younger age groups. Models were run using several categorization schemes, and ultimately, we chose a dichotomous cut-point of 65 years. The age-stratified models are presented in Table 2.

The final model for $<65$ years $(n=15,802)$ had 21 predictors, including matching variables. The largest effect estimates were observed with Parkinson's disease (OR $=12.57,95 \%$ confidence interval $[\mathrm{CI}]=6.47-24.44)$; memory complaints $(\mathrm{OR}=6.75,95 \%$ $\mathrm{CI}=4.48-10.18)$; multiple sclerosis $(\mathrm{OR}=7.06,95 \% \mathrm{CI}=4.89$ 10.21); epilepsy ( $\mathrm{OR}=4.17,95 \% \mathrm{CI}=3.02-5.75)$; and schizophrenia (OR=3.32, 95\% CI=1.93-5.71). This model provided moderate discriminatory ability $(\mathrm{AUC}=0.76)$.

The final model for individuals aged $\geq 65$ years $(n=104,183)$ closely resembled the all-ages model, with 31 predictors. Of these, memory complaints ( $O R=5.67,95 \% C I=5.09-6.33$ ); Parkinson's disease $(\mathrm{OR}=4.16,95 \% \mathrm{CI}=3.67-4.71)$; schizophrenia $(\mathrm{OR}=3.63,95 \% \mathrm{CI}=2.67-4.95)$; and bipolar disorder $(\mathrm{OR}=2.55,95 \% \mathrm{CI}=2.10-3.11)$ had the largest effect estimates. Discriminatory ability was poor $(\mathrm{AUC}=0.63)$.

Validation of the all ages and stratified models did not result in changes in discriminatory ability. Additional sensitivity analyses were conducted using a higher $P$ value $(0.05)$ for the stepwise selection process, which increased the number of variables in the models but did not improve discriminatory ability. The models were also run in the Alzheimer's group, MCI group, and drug-only group, but these models had poorer discriminatory ability.

\section{Discussion}

Using a nationally representative commercially insured and Medicare Advantage population, we created regression models to estimate the odds of a diagnosis of dementia in 3 years. Information available through administrative claims was used so that the algorithm was logistically practical for low-burden implementation for a larger provider, such as an integrated delivery system or insurance plan level. Best accuracy was observed among individuals aged $<65$ years.

Several factors associated with dementia in our models suggest that symptoms may already be present 3 years before clinical diagnosis. This may have been especially true in the older age groups, decreasing the discriminatory ability of our model. For example, memory complaints were strong predictors of case status and had the largest odds ratio in the model for individuals aged $\geq 65$ years. Epilepsy was also a strong predictor of case status among those aged $<65$. Seizures are a complication of Alzheimer's disease, even though epilepsy has not been included in predictive models previously. ${ }^{21}$ Similarly, Parkinson's disease was another strong predictor of case 
Predicting Diagnosis of Alzheimer's Disease and Related Dementias Using Administrative Claims

TABLE 1 Characteristics of Individuals Diagnosed with Dementia, 2011-2014, and Matched Controls at 2 Years Before Diagnosis ${ }^{a}$

\begin{tabular}{|c|c|c|c|c|c|c|c|}
\hline \multirow{2}{*}{\multicolumn{2}{|c|}{ Dementia categories, $\mathrm{n}(\%)$}} & $\begin{array}{c}\text { Controls } \\
(\mathrm{n}=95,464)\end{array}$ & \multirow{2}{*}{$\frac{P \text { Value }^{\mathrm{b}}}{\text { N/A }}$} & & $\begin{array}{c}\text { Cases } \\
(\mathbf{n}=24,521)\end{array}$ & $\begin{array}{c}\text { Controls } \\
(\mathrm{n}=95,464)\end{array}$ & \multirow{2}{*}{$\frac{P \text { Value }^{\mathrm{b}}}{<0.001}$} \\
\hline & & N/A & & \multicolumn{3}{|c|}{ Ancillary services, ${ }^{\mathrm{d}}$ events per year } & \\
\hline Alzheimer's disease & 2,531 (10.3) & & & 0 & $15,579 \quad(64)$ & $63,330 \quad(66)$ & \\
\hline Mild cognitive impairment & $3,748(15.3)$ & & & $>0$ & $8,942 \quad(36)$ & $32,134 \quad(34)$ & \\
\hline Lewy-body & $136(0.6)$ & & & Comorbidities, ${ }^{\mathrm{e}} \mathrm{n}(\%)$ & & & \\
\hline Fronto-temporal & $102(0.4)$ & & & Alcohol dependence and abuse & 167 & $348 \quad(<1)$ & $<0.001$ \\
\hline Vascular & $1,013 \quad(4.1)$ & & & Atrial fibrillation & $2,809 \quad(11)$ & $9,132 \quad(10)$ & $<0.001$ \\
\hline Nonspecific dementia & $10,046(41.0)$ & & & Anxiety (general) & $2,294 \quad(9)$ & $5,175 \quad(5)$ & $<0.001$ \\
\hline Drug only & $3,833(15.6)$ & & & Anemia & 2,064 & 5,858 & $<0.001$ \\
\hline Multi & $2,087(8.5)$ & & & Arthritis & $7,770 \quad(32)$ & $25,904 \quad(27)$ & $<0.001$ \\
\hline Multi (diagnosed by specialist) & $1,025 \quad(4.2)$ & & & Asthma & $1,602 \quad(7)$ & $5,275 \quad(6)$ & $<0.001$ \\
\hline $\begin{array}{l}\text { Age } 2 \text { years before diagnosis, }{ }^{c} \\
\text { mean (SD) }\end{array}$ & $74.9(11.5)$ & $74.9(11.5)$ & 0.470 & Bipolar disorder & $456 \quad(2)$ & $323(<1)$ & $<0.001$ \\
\hline \multirow{2}{*}{\multicolumn{3}{|c|}{ Age 2 years before diagnosis, years, $\mathrm{n}(\%)$}} & & Cancer & $3,799 \quad(15)$ & $15,427 \quad(16)$ & 0.01 \\
\hline$<45$ & & & 0.990 & Cataracts & $6,313 \quad(26)$ & $24,590 \quad(26)$ & 0.97 \\
\hline $45-54$ & 710 & $\frac{2,+01}{2807}$ & & Chronic kidney disease & $3,535 \quad(14)$ & $11,110 \quad(12)$ & $<0.001$ \\
\hline $55-64$ & $1,887 \quad(8)$ & 7,390 & & Chronic liver disease & $429 \quad(2)$ & $844 \quad(1)$ & $<0.001$ \\
\hline $65-74$ & $4,338 \quad(18)$ & $16,942 \quad(18)$ & & COPD & $3,636 \quad(15)$ & $11,529 \quad(12)$ & $<0.001$ \\
\hline $75-84$ & $9,250 \quad(38)$ & $36,045 \quad(38)$ & & Delirium & $224 \quad(1)$ & $201 \quad(<1)$ & $<0.001$ \\
\hline$\geq 85$ & $7,729 \quad(32)$ & $29,879 \quad(31)$ & & Depression & $2,941 \quad(12)$ & $4,764 \quad(5)$ & $<0.001$ \\
\hline \multicolumn{3}{|l|}{ Sex, ${ }^{b}$ n (\%) } & 0.780 & Diabetes & $7,126 \quad(29)$ & $21,968 \quad(23)$ & $<0.001$ \\
\hline Male & $9,305 \quad(38)$ & $36,318 \quad(38)$ & & Epilepsy & 488 & $522 \quad(1)$ & $<0.001$ \\
\hline Female & $15,216 \quad(62)$ & $59,146 \quad(62)$ & & Heart failure & $2,513 \quad(10)$ & 7,042 & $<0.001$ \\
\hline \multicolumn{3}{|l|}{ Census, ${ }^{\mathrm{c}} \mathrm{n}(\%)$} & 0.900 & Hip fracture & 366 & 882 & $<0.001$ \\
\hline Northeast & $2,899 \quad(12)$ & $11,273 \quad(12)$ & & Hyperlipidemia & $14,765 \quad(60)$ & 56,217 & 0.002 \\
\hline South & $10,251 \quad(42)$ & $39,669 \quad(42)$ & & Hypertension & $71,241 \quad(70)$ & 63,004 & $<0.001$ \\
\hline Midwest & $8,503 \quad(35)$ & $33,272 \quad(35)$ & & Injury & 6,679 & $19,442 \quad(20)$ & $<0.001$ \\
\hline West & $2,868 \quad(12)$ & $11,250 \quad(12)$ & & Ischemic heart disease & 5,912 & 19,088 & $<0.001$ \\
\hline \multicolumn{3}{|l|}{ Insurance, $\mathbf{n}(\%)$} & $<0.001$ & Multiple sclerosis & 229 & $158 \quad(<1)$ & $<0.001$ \\
\hline Commercial & $6,476 \quad(26)$ & $31,263 \quad(33)$ & & Obstructive sleep apnea & 2,698 & $6,932 \quad(7)$ & $<0.001$ \\
\hline Medicare & $18,045 \quad(74)$ & $64,201 \quad(67)$ & & Other anxiety disorders & 709 & 1,132 & $<0.001$ \\
\hline \multicolumn{4}{|c|}{ All-cause health care utilization, $\mathrm{n}(\%)$} & Other psychosis & 545 & $438 \quad(<1)$ & $<0.001$ \\
\hline \multicolumn{3}{|c|}{ Any, ${ }^{\mathrm{d}}$ events per year } & $<0.001$ & Parkinson's disease & 706 & $495 \quad(1)$ & $<0.001$ \\
\hline$<6$ & $5,290 \quad(22)$ & $28,927 \quad(30)$ & & Pneumonia & 1,370 & 3,705 & $<0.001$ \\
\hline $6-11$ & $5,339 \quad(22)$ & $23,530 \quad(25)$ & & Schizophrenia & 205 & $95(<1)$ & $<0.001$ \\
\hline $12-21$ & $5,909 \quad(24)$ & $22,057 \quad(23)$ & & Sepsis & 242 & $523 \quad(1)$ & $<0.001$ \\
\hline$>21$ & $7,983 \quad(33)$ & $20,950 \quad(22)$ & & Stroke & 3,352 & 8,377 & $<0.001$ \\
\hline \multicolumn{3}{|l|}{ Hospitalization, ${ }^{\mathrm{d}}$ events per year } & $<0.001$ & Substance abuse and dependence & 997 & 2,689 & $<0.001$ \\
\hline 0 & $20,258 \quad(83)$ & $83,812 \quad(88)$ & & Traumatic brain injury & 924 & $1,598 \quad(2)$ & $<0.001$ \\
\hline$>0$ & $4,263 \quad(17)$ & $11,652 \quad(12)$ & & Urinary tract infection & $4,132 \quad(17)$ & $11,702 \quad(12)$ & $<0.001$ \\
\hline \multicolumn{3}{|l|}{ Long-term care, ${ }^{\mathrm{d}}$ events per year } & $<0.001$ & \multicolumn{4}{|l|}{ Symptoms, ${ }^{\mathrm{e}}$ n (\%) } \\
\hline 0 & $22,627 \quad(92)$ & $92,557 \quad(97)$ & & Coordination difficulty & 575 & 842 & $<0.001$ \\
\hline$>0$ & $1,894 \quad(8)$ & $2,907 \quad(3)$ & & Confusion & $35(<1)$ & $24 \quad(<1)$ & $<0.001$ \\
\hline \multicolumn{3}{|l|}{ Outpatient, ${ }^{\mathrm{d}}$ events per year } & $<0.001$ & Dizziness & $2,901 \quad(12)$ & $7,454 \quad(8)$ & $<0.001$ \\
\hline$<5$ & $5,323 \quad(22)$ & $28,226 \quad(30)$ & & Falls & $514 \quad(2)$ & 781 & $<0.001$ \\
\hline $5-9$ & $5,176 \quad(21)$ & $22,397 \quad(23)$ & & Hearing loss & $1,666 \quad(24)$ & $22,855 \quad(20)$ & $<0.001$ \\
\hline $10-18$ & $6,214 \quad(25)$ & $23,323 \quad(24)$ & & Memory complaints & $1,079 \quad(4)$ & $594 \quad(<1)$ & $<0.001$ \\
\hline$>18$ & $7,808 \quad(32)$ & $21,518 \quad(23)$ & & Weight loss & 1,112 & $2,348 \quad(2)$ & $<0.001$ \\
\hline
\end{tabular}

$a_{N}=119,985$.

${ }^{b} P$ value from chi-square goodness of fit or Student's t-test.

cMatching variables.

${ }^{d}$ Events during the third year before diagnosis or matched index date for controls

${ }^{e}$ Comorbidities/symptoms present at end of third year before diagnosis or matched index date for controls.

$C O P D=$ chronic obstructive pulmonary disease; $N A=$ not applicable; $S D=$ standard deviation 


\begin{tabular}{|c|c|c|}
\hline & $\begin{array}{c}\text { Aged }^{\text {< }}<65 \text { Years }^{\mathrm{c}} \\
(\mathrm{n}=15,802)\end{array}$ & $\begin{array}{c}\text { Aged } \geq 65 \text { Years }^{\mathrm{d}} \\
(\mathrm{n}=104,183)\end{array}$ \\
\hline Age at diagnosis ${ }^{\mathrm{e}}$ & $0.98 \quad(0.98-0.98)$ & $1.00 \quad(0.99-1.00)$ \\
\hline Sex & $0.77 \quad(0.71-0.85)$ & $0.93(0.90-0.96)$ \\
\hline \multicolumn{3}{|l|}{ Censuse } \\
\hline Northeast & Reference & Reference \\
\hline South & $1.00 \quad(0.87-1.15)$ & $1.06 \quad(1.01-1.12)$ \\
\hline Midwest & $1.07 \quad(0.91-1.25)$ & $1.03(0.98-1.08)$ \\
\hline West & $1.03(0.87-1.23)$ & $1.12(1.05-1.20)$ \\
\hline \multicolumn{3}{|l|}{ Insurance } \\
\hline Commercial & Reference & Reference \\
\hline Medicare & $2.67(2.33-3.06)$ & $1.32(1.27-1.38)$ \\
\hline \multicolumn{3}{|c|}{ All-cause health care utilization } \\
\hline \multicolumn{3}{|c|}{ Any ${ }^{\mathrm{f}}$ events per year } \\
\hline$<6$ & Reference & Reference \\
\hline $6-11$ & $1.63(1.44-1.84)$ & $1.03(0.99-1.08)$ \\
\hline $12-21$ & $2.08(1.82-2.38)$ & $1.08(1.03-1.14)$ \\
\hline$>21$ & $3.16(2.75-3.63)$ & $1.19(1.13-1.26)$ \\
\hline \multicolumn{3}{|c|}{ Hospitalization, ${ }^{\mathrm{f}}$ events per year } \\
\hline 0 & & Reference \\
\hline$>0$ & & $0.86(0.82-0.90)$ \\
\hline \multicolumn{3}{|c|}{ Long-term care, ${ }^{\mathrm{f}}$ events per year } \\
\hline 0 & & Reference \\
\hline$>0$ & & $1.62(1.51-1.75)$ \\
\hline \multicolumn{3}{|c|}{ Outpatient, ${ }^{\mathrm{f}}$ events per year } \\
\hline$<5$ & Reference & \\
\hline $5-9$ & $1.49(1.24-1.80)$ & \\
\hline $10-18$ & $1.64(1.29-2.10)$ & \\
\hline$>18$ & $1.80(1.32-2.42)$ & \\
\hline \multicolumn{3}{|c|}{ Ancillary services,${ }^{f}$ events per year } \\
\hline 0 & Reference & \\
\hline$>0$ & $1.16(1.05-1.28)$ & \\
\hline
\end{tabular}

\begin{tabular}{|c|c|c|}
\hline & $\begin{array}{c}\text { Aged }<65 \text { Years }^{\mathrm{c}} \\
(\mathrm{n}=15,802)\end{array}$ & $\begin{array}{c}\text { Aged }^{2} \geq 65 \text { Years }^{\mathrm{d}} \\
(\mathrm{n}=104,183)\end{array}$ \\
\hline \multicolumn{3}{|l|}{ Comorbiditiesg } \\
\hline Anxiety & $1.49 \quad(1.29-1.73)$ & $1.18 \quad(1.11-1.26)$ \\
\hline Bipolar disorder & $2.95(2.22-3.92)$ & $2.55(2.10-3.11)$ \\
\hline Cancer & & $0.87 \quad(0.83-0.91)$ \\
\hline Cataracts & & $0.94 \quad(0.91-0.98)$ \\
\hline Chronic liver disease & $1.79(1.20-2.68)$ & \\
\hline Delirium & & $1.55 \quad(1.25-1.94)$ \\
\hline Depression & $1.83(1.59-2.11)$ & $1.74 \quad(1.64-1.85)$ \\
\hline Diabetes & $1.36(1.20-1.53)$ & $1.23(1.19-1.27)$ \\
\hline Epilepsy & $4.17 \quad(3.02-5.75)$ & $1.89 \quad(1.62-2.21)$ \\
\hline Hyperlipidemia & & $0.87(0.84-0.90)$ \\
\hline Injury & & $1.13(1.08-1.17)$ \\
\hline Ischemic heart disease & & $1.10(1.05-1.14)$ \\
\hline Multiple sclerosis & $7.06 \quad(4.89-10.21)$ & $2.39 \quad(1.78-3.20)$ \\
\hline Obstructive sleep apnea & $1.34 \quad(1.17-1.53)$ & $1.13 \quad(1.07-1.19)$ \\
\hline Other psychosis & & $2.08 \quad(1.79-2.41)$ \\
\hline Parkinson's disease & $12.57 \quad(6.47-24.44)$ & $4.16 \quad(3.67-4.71)$ \\
\hline Schizophrenia & $3.32(1.93-5.71)$ & $3.63 \quad(2.67-4.95)$ \\
\hline Stroke & $2.21 \quad(1.78-2.75)$ & $1.21 \quad(1.15-1.27)$ \\
\hline Traumatic brain injury & $1.63 \quad(1.17-2.28)$ & $1.27 \quad(1.14-1.40)$ \\
\hline Urinary tract infection & & $1.10 \quad(1.05-1.15)$ \\
\hline \multicolumn{3}{|l|}{ Symptomsg } \\
\hline Coordination difficulty & & $1.25 \quad(1.11-1.42)$ \\
\hline Dizziness & $1.47(1.23-1.75)$ & $1.20 \quad(1.14-1.26)$ \\
\hline Falls & & $1.33(1.18-1.51)$ \\
\hline Memory complaints & $6.75 \quad(4.48-10.18)$ & $5.67 \quad(5.09-6.33)$ \\
\hline Weight loss & & $1.47(1.36-1.59)$ \\
\hline
\end{tabular}

${ }^{a}$ Each model is adjusted for only those variables for which an estimate was presented. Variables chosen using stepwise selection $(P<0.001)$ with matching factors forced into model.

$b_{N}=119,985$

cArea under the curve $=0.76$.

${ }^{d}$ Area under the curve $=0.63$

eMatching variables.

EEvents during the third year before diagnosis or matched index date for controls.

gComorbidities/symptoms present at end of third year before diagnosis or matched index date for controls.

$C I=$ confidence interval; $O R=$ odds ratio.

status that is known to increase risk of dementia. ${ }^{22,23}$ Cognitive impairment is also common in multiple sclerosis, a strong predictor in our models. ${ }^{24}$ These comorbidities are associated with cognitive impairment or ADRD and provide additional evidence for delayed or missed diagnosis of dementia, which affects up to $50 \%$ of individuals with Alzheimer's disease. ${ }^{1,4}$ This misclassification of case status likely resulted in decreased accuracy of the models.

The accuracy of our claims-based model for individuals aged $<65$ years $(A U C=0.76)$ is similar to that of other published models ( $A U C=0.74-0.77$ ) that depended on primary data collected from patients. ${ }^{7-11}$ While our model predicts odds of dementia diagnosis within a relatively short, 3-year time frame, previous studies focused on prediction of dementia $10-40$ years in the future. ${ }^{9-11,25}$ The studies with the longest induction period (20 and 40 years) used baseline information obtained during midlife, similar to our model for those aged $<65$ years. ${ }^{10,11}$ Yet, despite moderate discriminatory ability, the utility of these models for initiating dementia screening or identifying individuals for clinical trials or targeted intervention is limited given the long lead time. A study with a shorter (6-year) induction period used data collected from 4 established cohorts of individuals aged $\geq 65$ years and reported variable accuracy (AUC range $=0.68-0.78$ ) depending on the cohort. ${ }^{7}$ This 
variability may have been related to the differing composition of each cohort. A study that used machine learning approaches to predict prevalent mild cognitive impairment reported lower discriminatory ability (AUC=0.69-0.73), although the investigators had access to lifestyle and demographic variables not available in claims. ${ }^{8}$ Thus, our results are better or consistent with previous studies, with efficiency and lower data collection burden and, therefore, easier to implement.

Consistent with findings from our study, diabetes, depression, and anxiety have been previously used to predict dementia diagnosis. ${ }^{7,910,12,13}$ In contrast, we also observed that bipolar and schizophrenia diagnoses, known risk factors for dementia, were strong predictors of dementia diagnosis. ${ }^{26,27}$

$\mathrm{HCU}$ variables were retained in the predictive models, although HCU was not a strong predictor of case status. Our hypothesis that cases would have higher HCU in the years leading up to dementia diagnosis was supported, but the association of increased HCU and dementia diagnosis was not strong. Nonetheless, the observed difference in HCU 3 years before dementia diagnosis emphasizes the need for earlier diagnosis and efforts to minimize HCU.

Mass cognitive screening of asymptomatic adults is not recommended by the United States Preventative Services Task Force. However, our algorithm could be considered for targeted screening of individuals who, according to the model, appear to be at increased risk. Our study did not test the use of the models in a system where results could be provided to clinicians for incorporation into care decisions. A useful next step would be to demonstrate feasibility and efficiency in a primary care setting. That is, to unobtrusively implement the model using claims data in a health care system to provide a message to clinicians, through an electronic medical record decisionsupport mechanism, suggesting screening only for those specific patients detected to be at high risk. This messaging could go to the primary care physician's office or to the pharmacy where a short screening tool could be used. The Alzheimer's Association has recommended short cognitive screeners for this purpose. ${ }^{5,6}$ These tools might be considered for anyone who the models suggest is at higher risk.

\section{Limitations}

There are several limitations that must be considered when interpreting results from this study. A case-control design was used that restricts calculation of risk or risk scores. Hence, the next step is to validate these algorithms in other cohorts. Administrative claims contain only diagnoses that are coded for reimbursement purposes and do not include results of neuropsychological testing for diagnosis confirmation or socioeconomic variables that could influence dementia risk. Nonetheless, predictive models that did have access to these variables performed only marginally better than ours. ${ }^{6-10}$
Most importantly, error in case ascertainment caused by delayed or incorrect diagnosis likely decreased the discriminatory ability of the model in older age groups. This hypothesis is supported by the large percentage of individuals in our cohort with nonspecific dementia (45\%), multiple dementia diagnoses (9\%), and individuals without a diagnosis who received antidementia medications (18\%). Further supporting this concept, factors selected into our models suggested that symptoms of dementia were already present 3 years before diagnosis. Validation studies in other cohorts are needed to hone the models and improve predictive ability.

Still, these models can target cognitive screening toward individuals at increased risk of dementia. Targeted screening can be performed rapidly in the primary care setting using one of the cognitive screens recommended by the National Institutes of Health or the Alzheimer's Association. ${ }^{6,28}$ Targeted screening could promote earlier dementia diagnosis, resulting in improved preparation and reduced HCU.1,3

\section{Conclusions}

We created age-stratified predictive models for earlier diagnosis of dementia using information available in administrative claims. These models have promise, particularly in the population aged $<65$ years, because of their efficiency, since they rely only on claims data. Future evaluation of the models should examine their efficiency and effectiveness in a clinical setting to promote targeted cognitive screening and earlier dementia recognition for specific individuals determined to be at risk. As technology evolves to provide access to patient-provided and/or genetic information, additional variables may improve predictive ability while maintaining efficiency.

\section{Authors}

JENNIFER S. ALBRECHT, PhD, University of Maryland School of Medicine, Baltimore. MAYA HANNA, MPH, and DURE KIM, PharmD, University of Maryland School of Pharmacy, Baltimore. ELEANOR M. PERFETTO, PhD, MS, University of Maryland School of Pharmacy, Baltimore; National Health Council, Washington, DC; and OptumLabs, Cambridge, Massachusetts.

AUTHOR CORRESPONDENCE: Jennifer S. Albrecht, PhD, Department of Epidemiology and Public Health, University of Maryland School of Medicine, 10 S. Pine St., MSTF 334C, Baltimore, MD 21201. Tel.: 410.706.0071; E-mail: jalbrecht@som.umaryland.edu. 


\section{DISCLOSURES}

This research was supported by AstraZeneca, Global CEO Initiative, Janssen, OptumLabs, and Roche. Albrecht was supported by Agency for Healthcare Quality and Research grant K01HS024560. Perfetto is employed by the National Health Council, which accepts membership dues and sponsorships from a variety of organizations and companies. The authors declare no other potential conflicts of interest.

\section{ACKNOWLEDGMENTS}

The authors thank members of the OptumLabs Alzheimer's constellation technical expert panel for guidance throughout this study.

\section{REFERENCES}

1. Alzheimer's Association. 2017 Alzheimer's disease facts and figures. Alzheimers Dement. 2017;13:325-73.

2. Hebert LE, Beckett LA, Scherr PA, Evans DA. Annual incidence of Alzheimer disease in the United States projected to the years 2000 through 2050. Alzheimer Dis Assoc Disord. 2001;15(4):169-73.

3. Alzheimer's Disease International. World Alzheimer report 2011. The benefits of early diagnosis and intervention. September 2011. Available at: https:// www.alz.co.uk/research/world-report-2011. Accessed September 8, 2018.

4. Bradford A, Kunik ME, Schulz P, Williams SP, Singh H. Missed and delayed diagnosis of dementia in primary care: prevalence and contributing factors. Alzheimer Dis Assoc Disord. 2009;23(4):306-14.

5. The Medicare Learning Network. The ABCs of the annual wellness visit. April 2017. Available at: https://www.cms.gov/Outreach-and-Education/ Medicare-Learning-Network-MLN/MLNProducts/downloads/AWV_chart ICN905706.pdf. Accessed September 8, 2018.

6. Cordell CB, Borson S, Boustani M, et al.; Medicare Detection of Cognitive Impairment Workgroup. Alzheimer's Association recommendations for operationalizing the detection of cognitive impairment during the Medicare Annual Wellness Visit in a primary care setting. Alzheimers Dement. 2013;9(2):141-50

7. Barnes DE, Beiser AS, Lee A, et al. Development and validation of a brief dementia screening indicator for primary care. Alzheimers Dement. 2014;10(6):656-65

8. Byeon H. A prediction model for mild cognitive impairment using random forests. Int J Adv Comput Sci App. 2015;6(12):8-12.

9. Exalto LG, Biessels GJ, Karter AJ, et al. Risk score for prediction of 10 year dementia risk in individuals with type 2 diabetes: a cohort study. Lancet Diabetes Endocrinol. 2013;1(3):183-90.

10. Exalto LG, Quesenberry CP, Barnes D, et al. Midlife risk score for the prediction of dementia four decades later. Alzheimers Dement. 2014;10(5):562-70.

11. Kivipelto M, Ngandu T, Laatikainen T, et al. Risk score for the prediction of dementia risk in 20 years among middle aged people: a longitudinal, population-based study. Lancet Neurol. 2006;5(9):735-41.
12. Pankratz VS, Roberts RO, Mielke MM, et al. Predicting the risk of mild cognitive impairment in the Mayo Clinic Study of Aging. Neurology. 2015;84(14):1433-42.

13. Reitz C, Tang M, Schupf N, et al. A summary risk score for the prediction of Alzheimer disease in elderly persons. Arch Neurol. 2010;67(7):835-41.

14. Albert SM, Glied S, Andrews H, et al. Primary care expenditures before the onset of Alzheimer's disease. Neurology. 2002;59(4):573-78.

15. Chen L, Reed C, Happich M, et al. Health care resource utilisation in primary care prior to and after a diagnosis of Alzheimer's disease: a retrospective, matched case-control study in the United Kingdom. BMC Geriatr. 2014;14:76-85.

16. Geldmacher DS, Kirson NY, Birnbaum HG, et al. Pre-diagnosis excess acute care costs in Alzheimer's patients among a U.S. Medicaid population. Appl Health Econ Health Policy. 2013;11:407-13.

17. Cho K, Gagnon DR, Driver JA, et al. Dementia coding, workup, and treatment in the VA New England Healthcare System. Int J Alzheimers Dis. 2014;2014:821894

18. Butler D, Kowall NW, Lawler E, et al. Underuse of diagnostic codes for specific dementias in the Veterans Affairs New England healthcare system. J Am Geriatr Soc. 2012;60(5):910-15.

19. Chung SD, Liu SP, Sheu JJ, et al. Increased healthcare service utilizations for patients with dementia: a population-based study. PLoS One. 2014;9(8):e105789.

20. Hill JW, Futterman R, Duttagupta S, et al. Alzheimer's disease and related dementias increase costs of comorbidities in managed Medicare. Neurology. 2002;58(1):62-70.

21. Larner AJ. Epileptic seizures in AD patients. Neuromolecular Med. 2010;12(1):71-77.

22. Levy G, Schupf N, Tang MX, et al. Combined effect of age and severity on the risk of dementia in Parkinson's disease. Ann Neurol. 2002;51:722-29.

23. Aarsland D, Andersen K, Larsen JP, et al. Risk of dementia in Parkinson's disease: a community-based, prospective study. Neurology. 2001;56(6):730-36. 24. Chiaravalloti ND, DeLuca J. Cognitive impairment in multiple sclerosis. Lancet Neurol. 2008;7(12):1139-51.

25. Jacqmin-Gadda H, Blanche P, Chary E, et al. Prognostic score for predicting risk of dementia over 10 years while accounting for competing risk of death. Am J Epidemiol. 2014;180(8):790-98.

26. Diniz BS, Teixeira AL, Cao F, et al. History of bipolar disorder and the risk of dementia: a systematic review and meta-analysis. Am J Geriatr Psychiatry. 2017;25(4):357-62.

27. Ribe AR, Laursen TM, Charles M, et al. Long-term risk of dementia in persons with schizophrenia: a Danish population-based cohort study. JAMA Psychiatry. 2015;72(11):1095-101.

28. HealthMeasures. NIH Toolbox for the Assessment of Neurological and Behavioral Function. U.S. Department of Health and Human Services.

Available at: http://www.healthmeasures.net/explore-measurement-systems/ nih-toolbox. Accessed September 8, 2018. 